\title{
Cellular and Developmental Strategies Aimed at Kidney Tissue Engineering
}

\author{
Gleb Martovetsky a, banjay K. Nigam ${ }^{a-d}$ \\ Departments of a Pediatrics, ${ }^{b}$ Biomedical Sciences, ${ }^{c}$ Medicine and ${ }^{\mathrm{d}}$ Cellular and Molecular Medicine, University of \\ California at San Diego, La Jolla, Calif., USA
}

\section{Key Words \\ Kidney · Tissue engineering · Regenerative medicine · Differentiation - Transdifferentiation · Progenitor cells · Organoid · Stem cell · Ureteric bud · Metanephric mesenchyme $\cdot$ Bioreactor}

\begin{abstract}
Background: With the rate of kidney disease on the rise, and a serious imbalance between the number of patients requiring a kidney transplant and the number of available donor kidneys, it is becoming increasingly important to develop alternative strategies to restore organ function to diminish the need for human donors. Summary: We review the current progress and future directions of a subset of these strategies which are ultimately aimed towards bioengineering a functional, implantable, kidney-like tissue construct or organoid that might be genetically matched to the patient. Key Messages: By combining the knowledge about normal kidney development with the rapidly growing knowledge in the field of cell differentiation and transdifferentiation, there is hope that partial or complete kidney function can be restored in patients with kidney disease - including genetic disorders, acute kidney injury, or chronic kidney disease with tissue-engineered construct(s).

(c) 2014 S. Karger AG, Basel
\end{abstract}

\section{Introduction}

In vivo, kidney development is considered to begin when the ureteric bud (UB) grows out from an epithelial tubule called the wolffian duct (WD) into a population of cells referred to as the metanephric mesenchyme (MM), which originates from the intermediate mesoderm. The UB and MM then go through mutually inductive interactions, where the MM supports the dichotomous branching of the UB to form the collecting duct system, while the UB induces the MM to undergo a complex process that gives rise to the proximal (including the epithelial cells of the glomerulus) through distal portions of nephrons. During this iterative process, vasculature and innervation originating from the base of the UB are also established. Once fully developed, the kidney contains more than two dozen distinct cell types which must be spatially organized to carry out their functions.

From a tissue-engineering perspective, while the ultimate endpoint is to create an organoid in a laboratory setting that can replace the function of a healthy kidney, the most feasible means of achieving this goal is not yet clear. A number of approaches have been proposed. With the advent of decellularized organ scaffolds, one conceivable option is to reintroduce the necessary types of dif-

\section{KARGER}

E-Mail karger@karger.com

www.karger.com/nee
(C) 2014 S. Karger AG, Basel

$1660-2129 / 14 / 1262-0101 \$ 39.50 / 0$
Sanjay K. Nigam

University of California at San Diego

9500 Gilman Drive, MC0693

La Jolla, CA 92093 (USA)

E-Mail snigam@ucsd.edu 
ferentiated patient-specific kidney cells into a full-size scaffold rather than following a strictly developmental program. This will require safe and efficient methods to program the distinct kidney cell types from patient cells. An alternative strategy is to mimic endogenous development, which consequently reduces the requirement of starting materials to a small set of progenitor cell populations, but this may necessitate a more intricate culture process. Nevertheless, multiple studies have demonstrated the self-contained ability of the UB and MM to instruct each other through kidney development, making the use of developmental approaches a promising possibility [1]. However, if the strategy is to mimic development from a set of progenitor cells, there is a still the question of whether a full-size engineered organ can be cultured in a lab setting and then implanted or whether it needs to undergo development in vivo.

\section{Lessons from Cell and Partial Organ Culture Systems Relevant to Renal Morphogenesis}

Pioneering studies carried out more than half a century ago by Grobstein [2] and colleagues demonstrated that after the UB and MM are specified these tissues can then be cultured in vitro and are capable of recapitulating much of the early kidney development - including branching morphogenesis of the collecting system and nephron formation - without major additional cues. Since this was discovered, a substantial amount of progress has been made in further deconstructing renal morphogenesis in vitro and in vivo in order to understand endogenous development as well as how these properties can be exploited to engineer functional renal tissue.

Because the UB and MM do not survive when cultured in isolation, efforts were directed at isolating the supportive and inductive cues provided by the two tissues to each other. To address this, immortalized cell lines of UB and $\mathrm{MM}$ origin were tested for their capacity to induce morphogenetic processes involved in kidney development. It was found that UB cell-conditioned medium, along with diffusion-limited signals [3] or added bFGF and TGFa [4], could induce nephrogenesis in isolated MM. Similarly, BSN cell (of MM origin)-conditioned media induced tubulogenesis of UB cells [5] and, in the presence of GDNF, of the isolated UB [6] when cultured in a 3-dimensional (3D) matrix. By purifying factors and testing them for the inductive capacity of the reciprocal tissue, specific combinations of growth factors were identified that could induce nephrogenesis in isolated MM [7] or branching morphogenesis of the isolated UB [8]. Since then, several other signaling pathways have been shown to have modulatory roles in the formation of the MMderived part of the nephron [9] and UB branching morpohogenesis [10].

It was also found that the WD could be induced to form buds in vitro [11]; this in vitro bud from the WD could replace the function of native UBs [12]. Furthermore, even an uninduced WD tubule was found to behave as the UB when recombined with MM [13]. Thus, the required starting materials for the collecting system of the kidney have been reduced to a tubular structure with a WD-like identity, presumably more attainable than the heterogeneous UB structure - which has a tip and stalk domain. Whichever strategy to engineer functional renal tissue proves to be the most efficient, the ability to dissect and modulate kidney organogenesis may be relevant to achieving success.

From a tissue-engineering viewpoint, the branching $\mathrm{UB}$ can be viewed as an iterative tip-stalk generator (ITSG)' $[14,15]$. This ability of the UB to act as an ITSG can be used to establish the architecture of the kidney; in other words, it could be used as the driver for organ engineering through recombination of tips with MM tissue $[16,17]$ or cells differentiated toward MM [14]. Importantly, the ITSG can be propagated for multiple generations by subculturing its tips, which then essentially act as new isolated UBs that can grow as the native UB (which can then be recombined with MM cells or tissue) (fig. 1). This is a robust strategy for generating functional renal organoids $[14,16]$. However, while relatively small implanted engineered renal constructs have been shown to recruit vasculature $[12,18]$, a larger one may require some type of preestablished vascular network.

The issue of vascularization aside, one of the major obstacles in translating tissue culture methods based on developmental processes is the lack of protocols to safely and efficiently reprogram patient-specific cells into the minimal set of required cell lineages that can undergo normal kidney development. Based on modified organ and cell culture findings, these appear to be cells that make up the UB (or possibly WD) and MM. It has been demonstrated that when early developing kidneys - consisting of the UB and $\mathrm{MM}$ - are enzymatically disassociated, reaggregated, and cultured they are able to reorganize into their respective domains and continue kidney development [19]. Furthermore, it has been shown that these constructs are able to develop vascularized nephrons when implanted under the renal capsule [20]. A remaining obstacle for this strategy is to establish the required progenitor tissues from cul- 


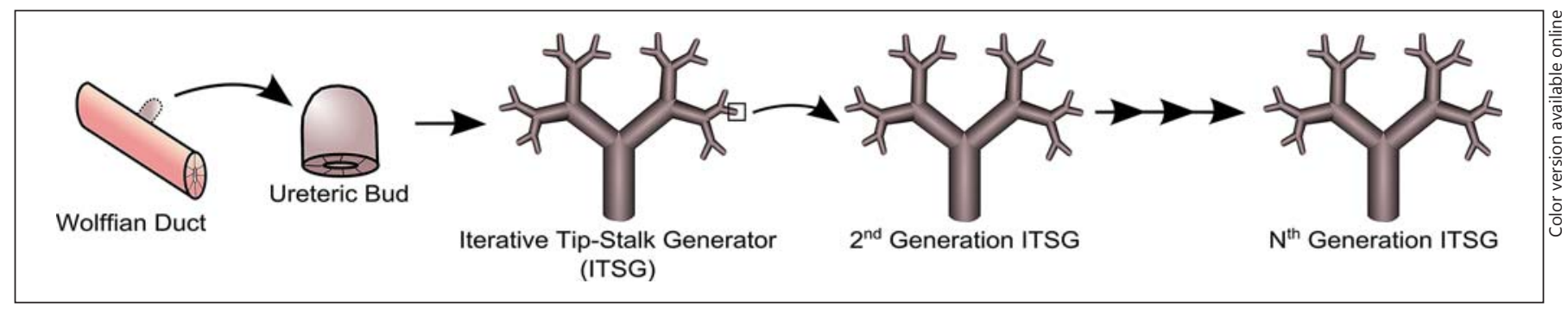

Fig. 1. Schematic of a possible strategy to engineer and propagate the renal collecting duct system as an ITSG. In vivo, the collecting duct system arises from the UB - an epithelial outgrowth that emerges from the WD upon induction by the MM. The UB is then further induced by the MM to undergo a finite number of dichotomous branching events (until the inducing MM is depleted), thus establishing the collecting system. This process can be recapitu- lated in vitro. First, an isolated WD is forced to bud by defined soluble factors. The resulting bud can then be dissected and induced to branch independently of the MM when cultured in a $3 \mathrm{D}$ matrix. Furthermore, tips from branching structures can then be dissected and induced to form branched structures for multiple generations, thereby serving as an ITSG. tured cells. While primary MM cells that are subcultured in 2 dimensions may lose some of their capacity to act as MM tissue, the fact that freshly prepared MM cell suspensions can behave as native MM upon reaggregation suggests that it may be possible to maintain this capacity over multiple cell divisions in culture given appropriate culture conditions [13]. This is further supported by the observation that, while the isolated MM cannot survive in culture without additional cues, MM cultured in the presence of FGF2 and BMP7 survived and retained the ability to undergo nephrogenesis for multiple days [21, 22]. Nevertheless, whether it is possible to establish 2 replenishable homogenous populations of cells that can be made to act as the $\mathrm{MM}$ and the $\mathrm{WD} / \mathrm{UB}$ from patient cells remains to be seen. If so, schemes have been proposed for engineering kidney tissue based on partial organ culture and cell culture methodologies $[12,13]$.

\section{Programming Renal Progenitor Cells}

A number of renal tissue-engineering strategies ultimately depend on the ability to establish patient-specific cells that are capable of being assembled into the initial kidney progenitor structures - the WD or UB and the MM (fig. 2). To explore methods to establish renal precursor cells, embryonic stem cells (ESCs) and induced pluripotent stem cells (iPSCs) have been used, the assumption being that many findings with ESCs are translatable to iPSCs. While it is not clear to what extent programmed cells can be used to fully recapitulate the behavior of either the UB or MM, multiple studies have

Cellular and Developmental Approaches

to Kidney Tissue Engineering reported differentiation of ESCs or iPSCs to renal progenitor-like cells according to marker expression and the capacity to integrate into developing kidneys $[23,24]$. Furthermore, recent studies have developed protocols that can differentiate pluripotent stem cells to renal progenitor cells that are capable of epithelialization and tubulogenesis in vitro (in the absence of native developing renal cells) [25-28]. Collectively, this work is an important step toward engineering renal tissue ex vivo from programmed cells alone.

Many of these studies have used various permutations of a small set of signaling molecules to induce renal marker expression in ESCs or iPSCs [mainly activin A, bone morphogenetic factors (BMP4 or BMP7), retinoic acid, fibroblast growth factors, WNT agonists, and leukemia inhibitory factor, all of which play important roles in early kidney development in vivo]. This supports the notion that endogenous development may serve as a guide for creating renal progenitors in vitro. However, it is important to determine what progenitor population would be most appropriate for renal tissue engineering. Many studies have shown expression of markers that demarcate the intermediate mesoderm, which is not solely committed to a renal fate, or markers that are found in, but not exclusive to, various renal cell lineages. Based on development, the target cell population should presumably replicate the functions of the MM, which is committed towards kidney lineages yet also sufficiently multipotent.

The functional contribution of cells differentiated in vitro from stem cells that have integrated into renal tissue remains to be clarified. Furthermore, stem cells have been reported to have the capacity to integrate into developing 


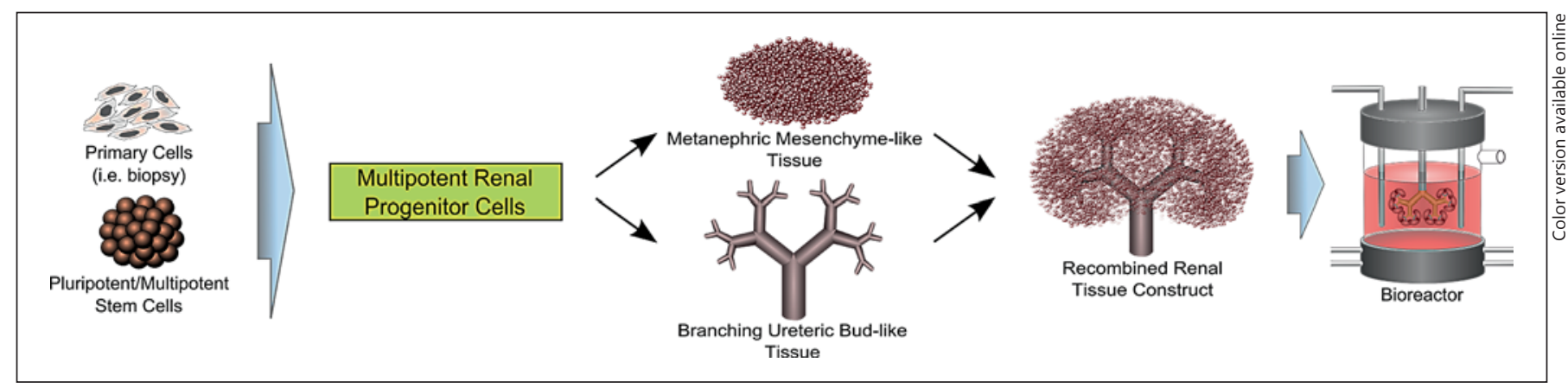

Fig. 2. Schematic of a modified organ culture approach to tissue engineering a kidney organoid from patient-derived cells. Potential cell sources include primary cells, adult stem cells (e.g. bone marrow-derived stem cells), or iPSCs. While differentiation towards expression of renal progenitor markers has been reported, the creation of cells that can recapitulate the endogenous capacity of the UB and MM remains hypothetical. Once this is achieved, the UB can be propagated in vitro (see fig. 1) and recombined with MM tissue to form a rudimentary renal organoid. While these resulting constructs are able to mature and become vascularized when implanted in vivo, initial maturation might be better achieved in a bioreactor prior to implantation or use in other applications.

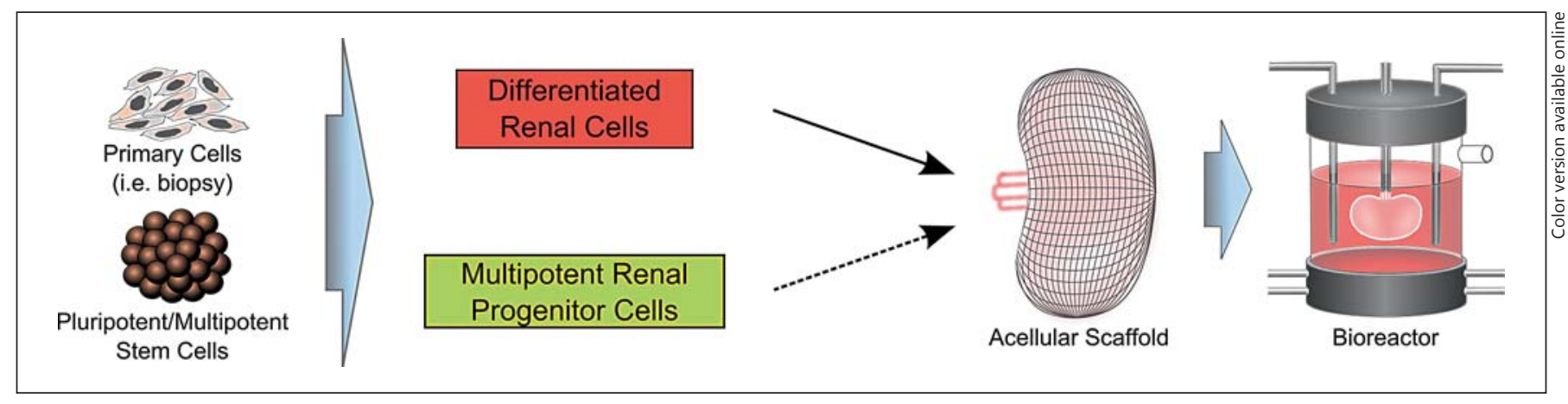

Fig. 3. Schematic of a cell-based approach to renal tissue engineering. Rather than following a developmental program, it may be possible to seed an acellular scaffold that recapitulates the renal architecture with patient-derived cells. Further development of this strategy will require the ability to establish the necessary dif- ferentiated renal cell types and/or renal progenitors from patient cells. Additionally, it may be important to refine the bioreactor design to be able to support the recellularization and maturation process of a relatively large organoid. renal tubules without any prior conditioning [29]. One more issue to consider is the fact that a true multipotent progenitor population would need to undergo multiple cell divisions, which could increase the potential for tumorigenicity. Thus, it is important to also consider the possibility of using patient-specific cells that are differentiated towards mature renal cell types.

\section{Programming Terminally Differentiated Renal Cells}

While a developmental approach is promising, an alternative strategy may be to generate full-sized implantable organs by recellularizing biocompatible scaffolds - which can be made by decellularizing cadaveric organs (likely pig or primate) or potentially by other means such as 3D bioprinting - with patient-derived cells (fig. 3). This was demonstrated in rats in a recent study, where rat kidneys were decellularized, recellularized with kidney and endothelial cells, and shown to carry out limited functions in vitro and in vivo [30]. As already mentioned, the success of this approach will largely depend on the ability to develop differentiation protocols for the multitude of mature renal cell types or potentially multipotent progenitors that can commit to the correct lineage based on their surrounding microenvironment.

In utero, the transition from a pluripotent state to a highly specialized, terminally differentiated cell type in- 
volves a myriad of spatiotemporal events. While perfectly recreating the endogenous developmental environment in vitro would seem ideal, this may be unrealistic. On the other hand, many studies have demonstrated that a small set of cues, whether it be via soluble signaling factors, reprogramming transcription factors, or other methods, are often sufficient to drive cells directly towards various cell lineages [31]. In some cases differentiation protocols involve several sequential steps attempting to mimic progressive developmental stages, but they do not fully correspond to the complexity of normal development. Nevertheless, these protocols have been able to achieve promising results, especially for nonrenal tissues such as the pancreas, liver, and cardiomyocytes, where cells programmed from either stem cells or somatic cells have been used to restore some functionality in injury/disease models. This provides hope that, with a deeper understanding of the lineage-determining traits of the different types of renal cells, it may become possible to develop methods to differentiate stem cells or transdifferentiate somatic cells towards cells capable of reconstituting a kidney.

Most of the early studies that have differentiated cells towards renal lineages have been aimed at creating a progenitor population. Recently, mature cell types have become a target of interest as well. While the field is far from being able to engineer all renal cell types, there are reports of establishing proximal tubule cells from iPSCs [32] and podocytes from ESCs [33]. Interestingly, while the endpoints appear to be morphologically and functionally different, both studies used retinoic acid, BMP7, and activin A to achieve the final phenotype; BMP2 was also included to differentiate towards a proximal tubulelike phenotype. Furthermore, these factors have also been used to program renal progenitor populations. It will be important to establish clear criteria to characterize the endpoint, as well as to test the specific capacity for which the cells are designed, such as function and integration, in an injury model or population of a scaffold.

While signaling factors have often been used to differentiate stem cells into various lineages, transcription factors are often considered to be mechanistically critical for cell lineage determination. Furthermore, while there are a number of reports of using soluble signaling factors to differentiate stem cells to a more restricted fate, most reports of changing the cellular fate from one differentiated state to another (transdifferentiation) have involved the introduction of transcription factors [31, 34, 35]. In a recent study, proximal tubule marker expression and

Cellular and Developmental Approaches

to Kidney Tissue Engineering organic anion transporter function (specific to proximal tubules in the kidney) were achieved by introducing two transcription factors - hepatocyte nuclear factors 1a and 4a (Hnf1a and Hnf4a) - via lentiviral transduction of mouse embryonic fibroblasts [36]. Specifically Hnf1a and Hnf4a were chosen because their binding motifs were highly enriched with p300-bound cis-regulatory enhancers in the kidney cortex (where proximal tubule cells are the major population). Genome-wide studies have revealed the importance of enhancers in regulating cell-specific expression. In fact, lineage-determining transcription factor motifs and corresponding factors have been found at enhancers in multiple cell lines, including 3 of the 4 'Yamanaka factors' in ESCs [37], suggesting that identifying enhancer-bound transcription factors is relevant for developing any desired cell type. However, it is important to consider that while Hnfla and Hnf4a were able to induce the expression of proximal tubule markers, including the drug transporter Oat1, these transcription factors, in combination with others, have also been used to establish the hepatocyte cell fate. Thus, additional cues are likely important for refining specificity.

The starting material (iPSCs, differentiated somatic cells, or adult stem cells) for reprogramming is also worth considering because the epigenetic memory of the initial cell state has been demonstrated to play a role in determining the endpoint cell phenotype. Nevertheless, in addition to the aforementioned studies, there are multiple reports of the successful establishment of differentiated cell types of multiple organs from various cell sources, indicating that this should be achievable for most lineages. Furthermore, strategies that require the establishment of terminally differentiated fates rather than multipotent progenitor cells may have a lower risk of tumorigenicity. Thus, it is important to expand the capability to establish mature cell types and explore their potential for renal tissue engineering.

\section{Conclusions}

Here we have offered a perspective on several options that may be developed to bioengineer implantable tissue capable of restoring kidney function. Most of the approaches to renal tissue engineering discussed here are limited by the ability to establish the required cell types, among other factors. Vascularization and scale-up remain important considerations. Furthermore, it is not clear whether it is better or easier to differentiate towards 
multiple terminal lineages or a smaller number of multipotent lineages that are capable of achieving the same terminal renal fates. With the many options that are being developed, the question appears to be not 'if' but 'when and how?'.

\section{Acknowledgment}

This work was partly supported by the National Institutes of Health National Institute of Child Health and Human Development (Grant U54-HD07160).

\section{References}

1 Nigam SK, Wu W, Bush KT: Organogenesis forum lecture: in vitro kidney development, tissue engineering and systems biology. Organogenesis 2008;4:137-143.

-2 Grobstein C: Inductive epitheliomesenchymal interaction in cultured organ rudiments of the mouse. Science 1953;118:52-55.

3 Barasch J, Pressler L, Connor J, et al: A ureteric bud cell line induces nephrogenesis in two steps by two distinct signals. Am J Physiol 1996;271:F50-F61.

4 Karavanova ID, Dove LF, Resau JH, et al: Conditioned medium from a rat ureteric bud cell line in combination with bFGF induces complete differentiation of isolated metanephric mesenchyme. Development 1996; 122:4159-4167.

5 Sakurai H, Barros EJ, Tsukamoto T, et al: An in vitro tubulogenesis system using cell lines derived from the embryonic kidney shows dependence on multiple soluble growth factors. Proc Natl Acad Sci USA 1997;94:6279-6284.

-6 Qiao J, Sakurai H, Nigam SK: Branching morphogenesis independent of mesenchymal-epithelial contact in the developing kidney. Proc Natl Acad Sci USA 1999;96:7330-7335.

7 Barasch J, Yang J, Ware CB, et al: Mesenchymal to epithelial conversion in rat metanephros is induced by LIF. Cell 1999;99:377-386.

8 Sakurai H, Bush KT, Nigam SK: Identification of pleiotrophin as a mesenchymal factor involved in ureteric bud branching morphogenesis. Development 2001;128:3283-3293.

-9 Rosines E, Schmidt HJ, Nigam SK: The effect of hyaluronic acid size and concentration on branching morphogenesis and tubule differentiation in developing kidney culture systems: potential applications to engineering of renal tissues. Biomaterials 2007;28:48064817.

10 Bush KT, Sakurai H, Steer DL, et al: TGF-beta superfamily members modulate growth, branching, shaping, and patterning of the ureteric bud. Dev Biol 2004;266:285-298.

11 Maeshima A, Vaughn DA, Choi Y, et al: Activin $\mathrm{A}$ is an endogenous inhibitor of ureteric bud outgrowth from the Wolffian duct. Dev Biol 2006;295:473-485.

12 Rosines E, Sampogna RV, Johkura K, et al: Staged in vitro reconstitution and implantation of engineered rat kidney tissue. Proc Natl Acad Sci USA 2007;104:20938-20943.
13 Rosines E, Johkura K, Zhang H, et al: Constructing kidney-like tissues from cells based on programs for organ development: toward a method of in vitro tissue engineering of the kidney. Tissue Eng Part A 2010;16:24412455

14 Nigam SK: Concise review: can the intrinsic power of branching morphogenesis be used for engineering epithelial tissues and organs? Stem Cells Transl Med 2013;2:993-1000.

15 Nigam SK, Shah MM: How does the ureteric bud branch? J Am Soc Nephrol 2009;20: 1465-1469.

16 Steer DL, Bush KT, Meyer TM, et al: A strategy for in vitro propagation of rat nephrons. Kidney Int 2002;62:1958-1965.

17 Steer DL, Nigam SK: Developmental approaches to kidney tissue engineering. Am J Physiol Renal Physiol 2004;286:F1-F7.

18 Hammerman MR: Transplantation of renal primordia: renal organogenesis. Pediatr Nephrol 2007;22:1991-1998.

19 Unbekandt M, Davies JA: Dissociation of embryonic kidneys followed by reaggregation allows the formation of renal tissues. Kidney Int 2010;77:407-416.

20 Xinaris C, Benedetti V, Rizzo P, et al: In vivo maturation of functional renal organoids formed from embryonic cell suspensions. J Am Soc Nephrol 2012;23:1857-1868.

21 Dudley AT, Godin RE, Robertson EJ: Interaction between FGF and BMP signaling pathways regulates development of metanephric mesenchyme. Genes Dev 1999;13:1601-1613.

22 Barasch J, Qiao J, McWilliams G, et al: Ureteric bud cells secrete multiple factors, including bFGF, which rescue renal progenitors from apoptosis. Am J Physiol 1997;273:F757F767.

23 Xia Y, Nivet E, Sancho-Martinez E, et al: Directed differentiation of human pluripotent cells to ureteric bud kidney progenitor-like cells. Nat Cell Biol 2013;15:1507-1515.

24 Takasato M, Maier B, Little MH: Recreating kidney progenitors from pluripotent cells. Pediatr Nephrol 2014;29:543-552.

25 Taguchi A, Kaku Y, Ohmori T, et al: Redefining the in vivo origin of metanephric nephron progenitors enables generation of complex kidney structures from pluripotent stem cells. Cell Stem Cell 2014;14:53-67.
26 Araoka T, Mae S, Kurose Y, et al: Efficient and rapid induction of human iPSCs/ESCs into nephrogenic intermediate mesoderm using small molecule-based differentiation methods. PLoS One 2014;9: e84881.

27 Lam AQ, Freedman BS, Morizane R, et al: Rapid and efficient differentiation of human pluripotent stem cells into intermediate mesoderm that forms tubules expressing kidney proximal tubular markers. J Am Soc Nephrol 2013, Epub ahead of print.

28 Takasato M, Er PX, Becroft M, et al: Directing human embryonic stem cell differentiation towards a renal lineage generates a self-organizing kidney. Nat Cell Biol 2014; 16:118-126.

29 Steenhard BM, Isom KS, Cazcarro P, et al: Integration of embryonic stem cells in metanephric kidney organ culture. J Am Soc Nephrol 2005;16:1623-1631.

30 Song JJ, Guyette JP, Gilpin SE, et al: Regeneration and experimental orthotopic transplantation of a bioengineered kidney. Nat Med 2013;19:646-651.

-31 Graf T, Enver T: Forcing cells to change lineages. Nature 2009;462:587-594.

-32 Narayanan K, Schumacher KM, Tasnim F, et al: Human embryonic stem cells differentiate into functional renal proximal tubular-like cells. Kidney Int 2013;83:593-603.

33 Song B, Smink AM, Jones CV, et al: The directed differentiation of human iPS cells into kidney podocytes. PLoS One 2012; 7:e46453.

34 Jopling C, Boue S, Izpisua Belmonte JC: Dedifferentiation, transdifferentiation and reprogramming: three routes to regeneration. Nat Rev Mol Cell Biol 2011;12:79-89.

35 Zaret KS, Carroll JS: Pioneer transcription factors: establishing competence for gene expression. Genes Dev 2011;25:2227-2241.

36 Martovetsky G, Tee JB, Nigam SK: Hepatocyte nuclear factors 4a and 1a (Hnf4a and Hnfla) regulate kidney developmental expression of drug-metabolizing enzymes and drug transporters. Mol Pharmacol 2013;84: 808-823.

37 Heinz S, Benner C, Spann N, et al: Simple combinations of lineage-determining transcription factors prime cis-regulatory elements required for macrophage and $\mathrm{B}$ cell identities. Mol Cell 2010;38:576-589. 